\title{
APPLYING THE COMPUTER AIDED SYSTEMS IN EDUCATION PROCESS
}

\author{
Miriam MATÚŠOVÁ, Erika HRUŠKOVÁ \\ Slovak University of Technology
}

\begin{abstract}
:
The article summarizes the experience of laboratory technical subjects teaching methodologies using a number of software products. The main aim is to modernize the teaching process in accordance with the requirements of today - based on information technology. Increasing of the study attractiveness and effectiveness is due to the introduction of Computer Aided (CA) technologies in the learning process. This paper discussed the areas where individual CA system used and concrete examples used in the education process. They are CAD system Catia V5, FluidSIM created and simulates the pneumatics and electropneumatic schemes and circles, software witness used for simulation of material flow planning and RobotStudio that illustrates operated Robot in his own manipulated area. Environment using CA systems are briefly presented in each chapter.
\end{abstract}

Key words: Education, Computer Aided (CA) systems, Computer Aided (CA) technologies, simulation

\section{INTRODUCTION}

The information society has brought benefits in addition to several problems. The amount of information continues to grow. The information amount needs to process in most professions. Absorbing the information is no longer dominant. Developing the ability to obtain relevant information, process them and draw the necessary conclusions is a priority. Knowledge acquired at school is still sufficient for a shorter period of time. The need for continuous lifelong learning is essential. The requirement to improve the quality and speed of acquisition of knowledge and skills are gaining prominence.

With the Computer Aided Design (CAD) technologies development and the CAD systems gradual adoption by industry, the responsibilities of maintaining, developing, and CAD systems supporting have transferred from manufacturing companies to software companies, through spin-off, merging, and reorganization. Students in universities want to learn technologies that can best serve their future careers. The criteria will vary from person to person, from professional to professional, and from job to job.

Implementation of CA system into education is realized in our laboratory. The aim is to increase the readiness of our graduates in engineering practice. Increase the efficiency and attractiveness of study is achieved. CA systems used in our laboratory are well used by manufacturing companies.

\section{CA SYSTEMS}

Since the beginning of CAD in the 1960s, CAD technologies have found their way into colleges and universities. Currently, in the colleges and universities, CAD technologies have been taught in different disciplines with different focuses [8]. Industrial applications are the driving forces for the development of CAD technologies and CAD systems. Early development of CAD systems had been backed by major manufacturing companies. During the past three decades, CAD systems have evolved from Computer Assisted Drawing/Drafting, to Computer Aided Design, and to a stage now called by most CAD software vendors: Product Lifecycle Management [3].

Commercial CAD systems' developers have focused mostly on adding functionality in their tool sets, rather than upon making those tools fundamentally more usable. CAD tools influence the engineer's ability to solve engineering problems both positively and negatively. CAD has come a very long way in assisting and supporting the product development process for products, particularly for those which would take considerable time to produce using manual processes [3].

CA systems are computer systems intended to support activities at all engineering stages - from development and design, production planning, to actual production and assembly, warehousing and shipping. These systems allow accelerate and simplify the particular modeling and drawing, dimensioning, perform analyzes design as well as various administrative activities such as archiving, searching and more. They are all modeling software and drawings, the technical computing, simulation and analysis. Using and knowledge of CA systems are commonplace each designer. The continuous training is necessary in this field [1].

Software products are used in several project phases. Students within the subjects solve as follows:

- Designs and creates 3D models of components,

- Creation of entire assemblies,

- Proposal production system, 
- Proposal production system control and its simulation,

- Designs and simulates manufacturing process.

\section{D AND 3D MODEL}

The CAD technologies may be taught as tools to assist design, drawing and drafting in engineering disciplines and consumer product design disciplines. Students can create or edit lines and model shapes quickly and accurately with accurate dimensions and scale in a CAD environment.

There are a variety of CAD environments ranging from knowledge-based to parametric, from surface modelling to physics based CAD, from mechanical (M-CAD), to medical CAD and from the common desktop-platform to virtual reality VR [3].

The designer who creates the definitive geometric data of a product should know some basic concepts of engineering in order to avoid major geometric flaws due to elementary mistakes in engineering [9]. Each engineer responsible for an aspect of a product should know how to adequately use a CAD-system. For a very complex part, a designer with super user status may be required to define its geometry. For difficult engineering analyses, subject matter experts may be involved. Concurrent work has to be carefully managed [4].

Students begin creating simple parts models with CATIA V5 support. In the next step, they design assemblies and provide them technical documentation generation from 3D Catia V5 environment. An example of such a mounting assembly is shown in Fig. 1.

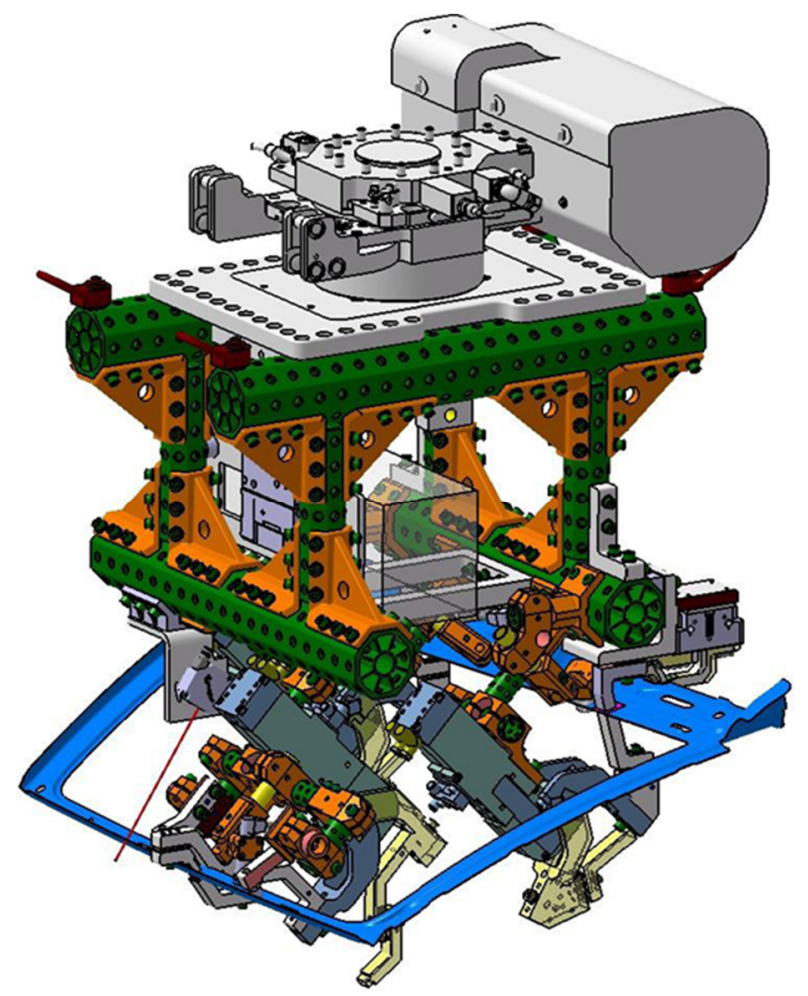

Fig. 1 3D model of robot gripper

Their next task is to design manufacturing system based on product. It already requires some engineering knowledge.
Students have to choose the right machines and propose assembly machines based on the technological process of production. The proposed manufacturing facilities properly deploy and propose automating material flows. Industrial robots, various conveyors or buffers may be used. Those are selected from the databases available on the market. The same, there is also necessary to take into account the properties of the used components material and the technology of their production. For example, the holes on the components made by a laser whose changes in parameters significantly affect the structure of the used materials [6]. Thus, the entire system compiled on the basis of the principle of modularity. Students design of some device and workstations are illustrated on Figs. 2 and 3. Both design models was created in CATIA environments.

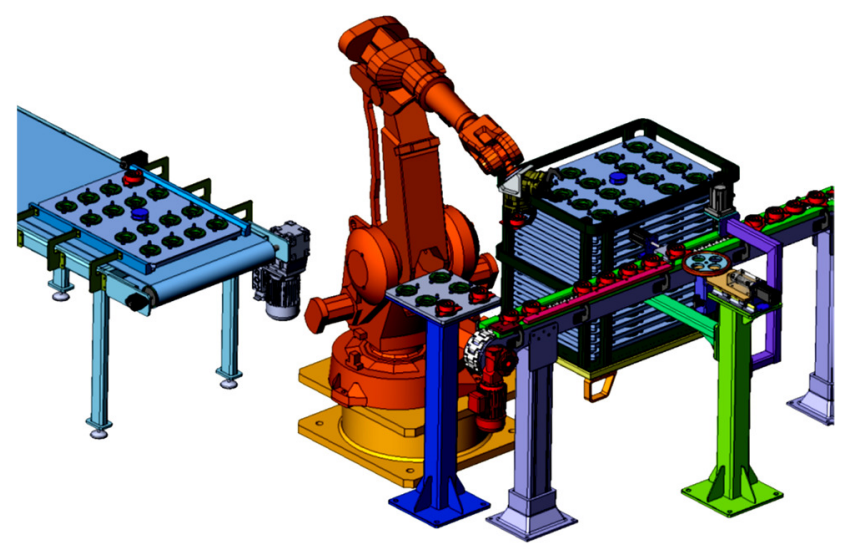

Fig. 2 3D model of workstation with angular robot

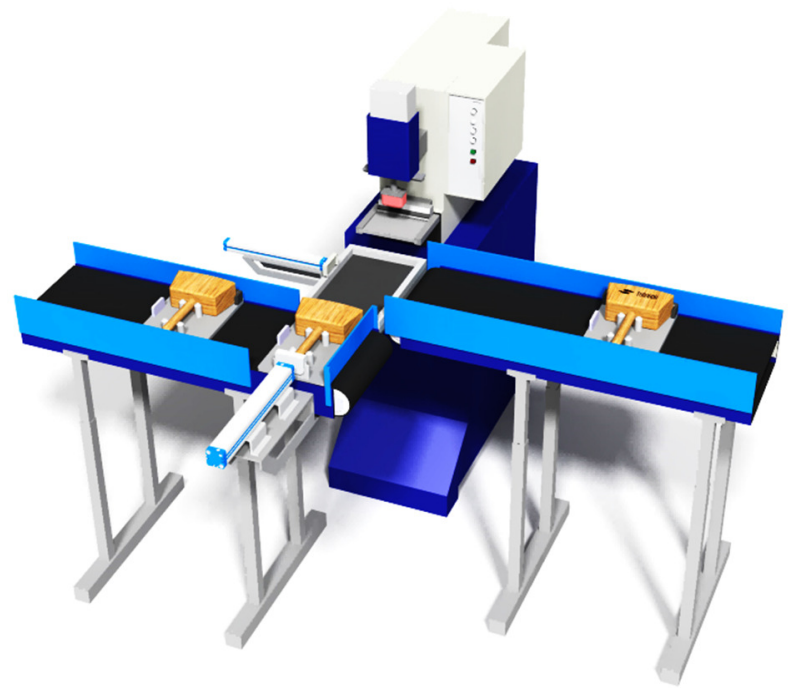

Fig. 3 Design of automated devices for labeling wooden hammers

\section{CONTROL SYSTEM SIMULATION}

Simulation allows you to quickly and easily understand the process and provides an enormous potential for finding reserves in the design process. As a tool for scheduling processes is advantageous in cases, predictive planning and decision-making is an alternative. Practice requires rapid tools to provide quality results Fig. 4 [2]. 


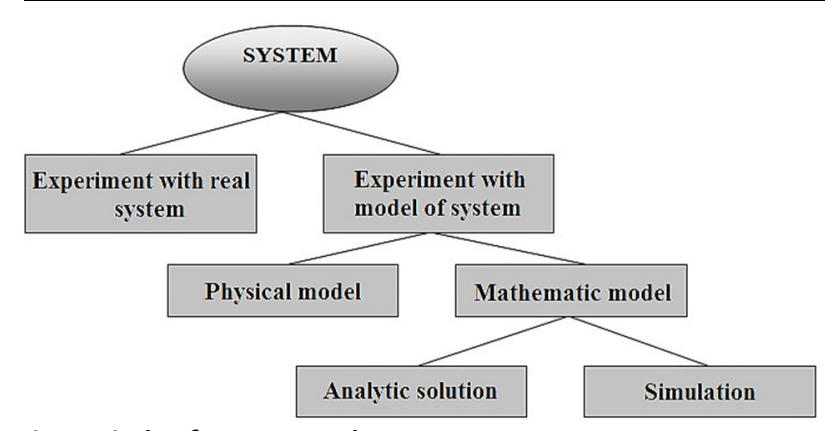

Fig. 4 Kinds of system study

System research by the experiment with the system model through the mathematical model with using mathematical expression. All of these parts are defined in the form of the parcial differential equations. These equations deals to the analytic resolution Fig. 5. Analytic resolution is possible to obtain by several methods:

- Methods of final differencions.

- Methods of weight residue.

- Reyleigh-Ritz methods.

- Monte Carlo method.

- Combination of methods.

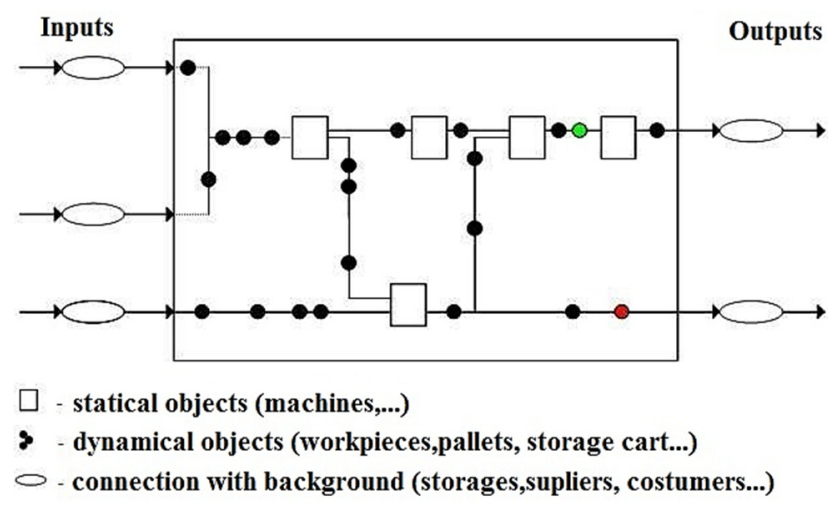

Fig. 5 Example mathematic model of the production system

System research realised through the system experiment with model by mathematical model is needed to verificate by simulation. There is monitoring that the mathematical model matching to system behaviour which is analysed. There is needed to establish the accuracy of asked model and if it is the designed solution enough accurate to get asked aim that the model is designed for. The compared results obtained by experiments may be different with regard to calculated result. These differences may be minimalised by modification of inputs factors or using of new ones. With modifications in researched model is necessary to analyse numerical solutions again, like the verificate the relevance [2].

With detail results of base steps of simulation project, there is necessary to define and next to proceed according to developed evolutional diagram [7]. This diagram except problem formulation includes:

- the aims,

- project plan,

- time plan,

- costs of project,

- design of conceptual model,

- identification of system elements,

- relations between them,

- the real inputs data and the conceptual model transformation into computer model Fig. 6.

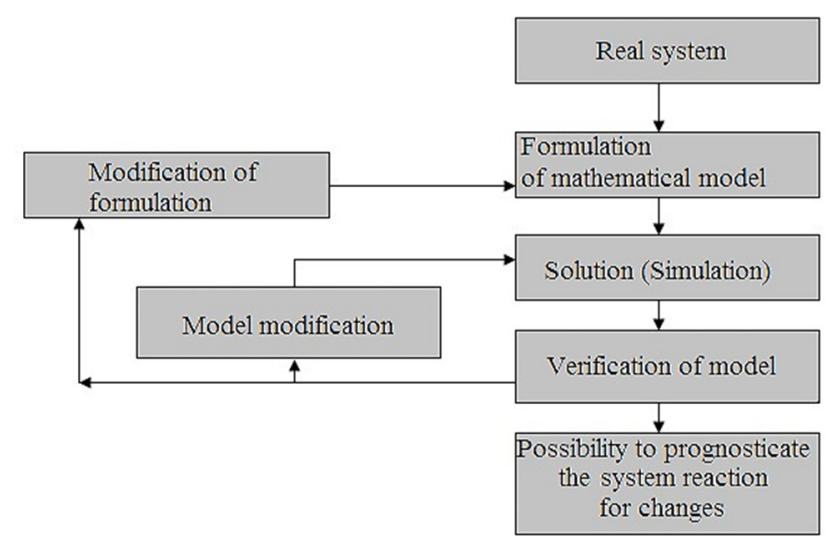

Fig. 6 Process control and model modification

Software FluidSIM was used as a simulation tool. FluidSIM 4 is comprehensive software for creation, simulation, instruction and study of pneumatic, electropneumatic, electrohydraulic and digital circuits [10]. Various integration processes are available to the simulator which are selected and controlled intelligently in relation to the operating time. Creation of control circuit is relatively simple and consists of:

- Selection of individual components of hierarchical libraries,

- Defining and setting the required values of individual components,

- Logical link between different components [1].

Fig. 7 illustrates an automated device control circuit establishment by FluidSIM environment. This software allows in addition to compiling the circuit simulation verify its functionality.

The next software used in our CAD laboratory is RobotStudio. This allows creating, simulating, optimizing and validating virtual robotic and automated manufacturing processes based on product configuration, data sources, and even in offline mode. 


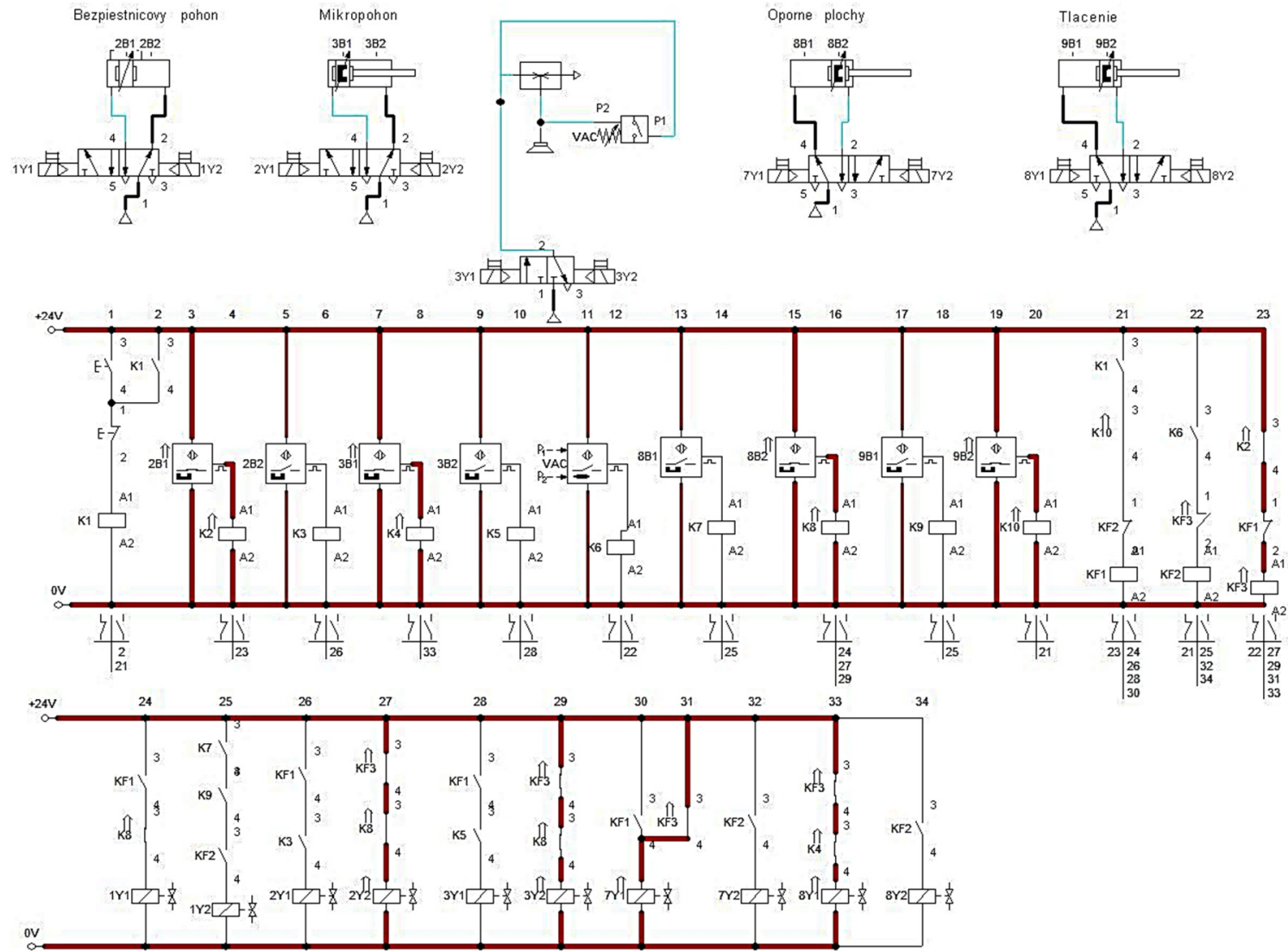

Fig. 7 Control circuit simulation by FluidSIM

\section{MANUFACTURING PROCESS PLANNING}

Simulation is one of the most frequently used techniques for the analysis and design of manufacturing systems, because it can save considerable time and money by viewing the dynamics of a complex manufacturing system, and provide insight into and a better understanding of those dynamics [1].

Students simulate manufacturing process planning by Witness software. Witness the world's leading simulation software used in dealing with production, service and logistics processes. Allows you to simulate the working environment, simulate consequences of different decisions and understand no matter how complicated the process even before implementation of proposed solutions. Managers to help minimize the risks changes in society and the result are greater trust management companies for new projects. Witness is system for creating interactive visual simulation model Fig. 8.

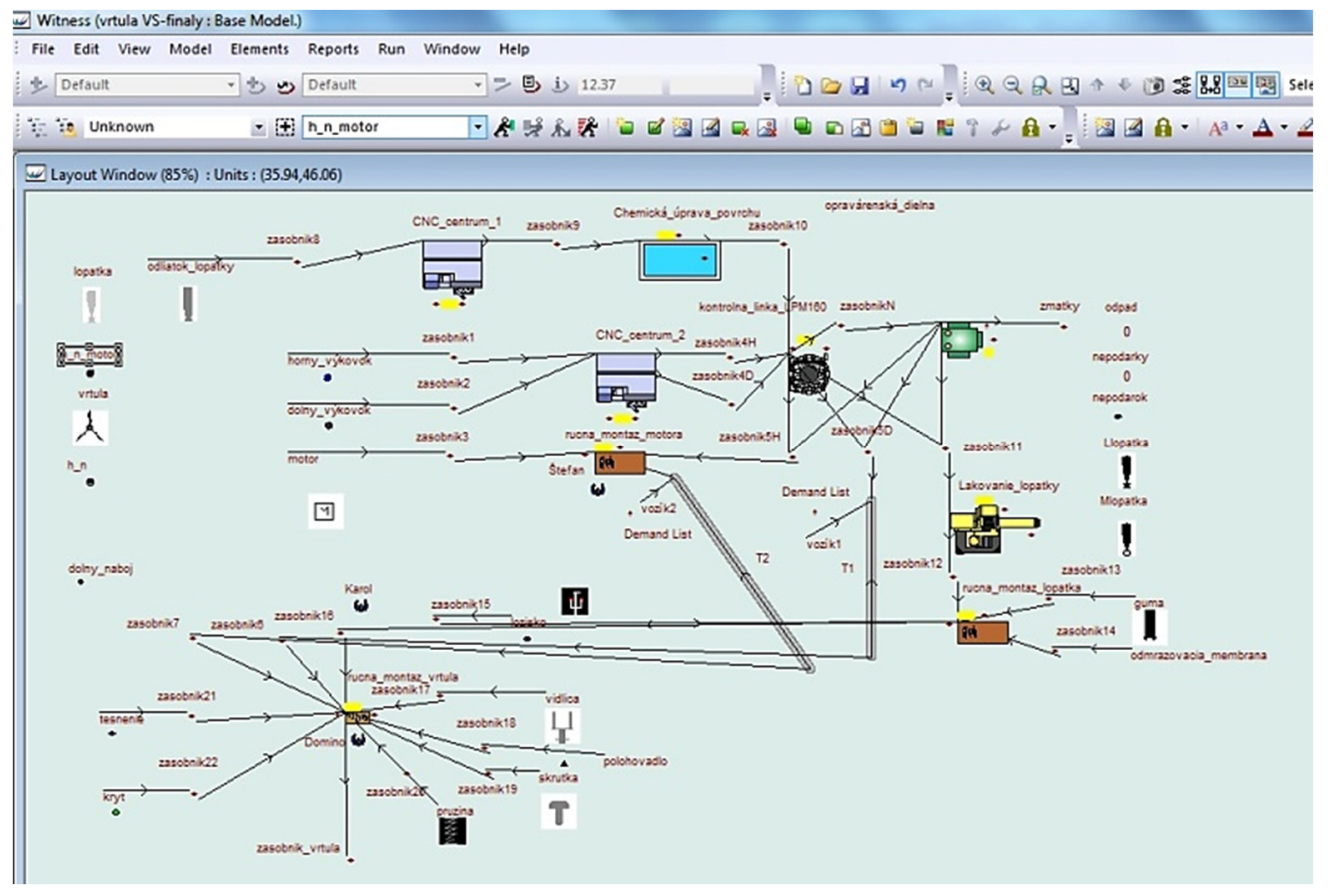


Dynamically displays movement and state of the elements (components) for the specified elements (reservoir, machine, conveyor, etc.) [5].

\section{CONCLUSION}

The main aim of the project is the establishment of laboratory with the group of training modules from the area of automation and industrial robotics, which will serve the several purposes. This laboratory will enable to develop students' knowledge and practical skills in the area of automated and robotic systems, applying the innovative educational program and methodology which use modern IT technologies, e-learning including. The aim is also an elaboration of study materials with widely applicable exercises that can make the laboratory study more effective and can serve the further knowledge and skills improvement for our graduates. There is the innovation designed to aim that increase the productivity of manufacture in dependence of production programs of factories. Flexible substitution of human to robot is designed in the several kinds of subsystems like production, logistics, storage, the maintenance, the control of data information transmission. Within designed modifications in production process, there are analysed the designs through the virtual industrialization that have to ensure the manufacture processes are modified and set to make the work in the real time.

\section{ACKNOWLEDGMENT}

The contribution is sponsored by the project 015STU4/2018 named Specialised laboratory supported by multimedia textbook for subject "Production systems design and operation" for STU Bratislava.

\section{REFERENCES}

[1] J. G. H. Carlson, A. C. Yao. "Simulating an agile, synchronized manufacturing system," International Journal of Production Economics, Vol. 112, pp. 714722, Apr. 2008.

[2] M. Matúšová. Application of simulation method in manufacturing system design. Trnava, STU Bratislava, 2015, pp. 88, ISBN 978-80-227-4487-4.

Ing. Miriam Matúšová, PhD., Ing. Erika Hrušková, PhD. Slovak University of Technology, Faculty of Material Science and Technology, Institute of Production System J. Bottu 24, 91724 Trnava, Slovak Republic e-mail: miriam.matusova@stuba.sk erika.hruskova@stuba.sk
[3] Z. Kosmadoudia, T. Lima, J. Ritchie, S. Louchart, Y. Liu, R. Sunga. Engineering design using game-enhanced CAD: The potential to augment the user experience with game elements". Computer-Aided Design, Vol.5, Issue 3, pp. 777-795, Mar. 2013.

[4] D. A. Field. "Education and training for CAD in the auto industry". Computer-Aided Design, vol. 36, issue 14, pp. 1431-1437, Dec. 2004.

[5] P. Važan, et. all.. Modeling and simulation of systems. Simulator Witness. Trnava, Alumni Press Trnava, 2012, ISBN 978-80-8096-160-2

[6] B. Šimeková, I. Kovaříková, E. Hodúlová. „Research of Cladded Layers Structures Changes Created by Laser Beam Technology Using a Wire Filler Material“, In Applied Mechanics and Materials: $3^{\text {rd }}$ International Conference on Computer-Aided Design, Manufacturing, Modeling and Simulation (CDMMS 2013), Chongqing, China, 21-23 September 2013. pp. 9-12. ISSN 1660-9336.

[7] B. Juhásová, M. Juhás, I. Halenár. „TCP/IP protocol utilisation in process of dynamic control of robotic cell according Industry 4.0 concept." In SAMI 2017, IEEE $15^{\text {th }}$ International Symposium on Applied Machine Intelligence and Informatics, Herl'any, Slovakia, 2017, pp. 000217-000221, ISBN 978-15090-5654-5.

[8] J. Kurnátová, P. Važan, G. Križanová, K. Orihelová, Katarína. „Assignment of labour to a production line depending on lot size". In Applied Mechanics and Materials: Novel Trends in Production Devices and Systems. Vol. 474 (2014), p. 121-126. ISSN 16609336.

[9] Š. Václav, P. Koštál, Š.Lecký, D. Michal, B. Bako. „Assembly system planning in automotive industry with use of discrete event simulation." In Proc.Vehicle and Automotive Engineering 2: proceedings of the 2nd VAE 2018, Miškolc, Hungary. 1. vyd. Cham: Springer, 2018, pp. 503-515. ISBN 9783-319-75676-9.

[10] K. Velíšek, P. Koštál. „Pneumatics and Electropneumatic Control Laboratory." In Proc. IC4E 2010: International Conference on e-Education, e-Business, e-Management and e-Learning. 2010, pp. 651-654. ISBN 978-0-7695-3948-5. 